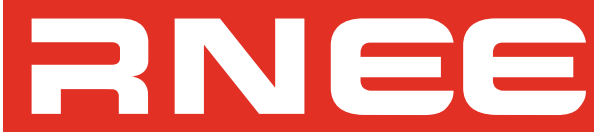

Revista Nicolaita de Estudios Económicos

Nueva Época

Instituto de Investigaciones Económicas y Empresariales
Universidad Michoacana de San Nicolás de Hidalgo

Volumen XVI, Número 2, Julio - Diciembre 2021, pp. 21 - 39

ISSN (Print): 1870 - 5464 ISSN (On-line): 2007 - 9877

DOI: $10.33110 /$ rnee.v16i2.327

(Recibido: 28/abril/2021; Aceptado: 30/junio/2021)

\title{
Impulso al desarrollo local de regiones y comunidades en situación de pobreza. Propuesta de planeación estratégica aplicada en una comunidad del sureste de México
}

\section{Boosting the local development of regions and communities in a situation of poverty. Strategic planning proposal applied in a community in southeastern Mexico}

\author{
José Félix García Rodríguez a * \\ Naamán Izquierdo Balcázar b \\ Lenin Martínez Pérez ${ }^{c}$ \\ Aída Armenta Ramírez d \\ José Ramón Contreras De la Cruz ${ }^{\mathrm{e}}$
}

\section{Resumen}

La planeación estratégica representa una alternativa viable para impulsar el desarrollo local y regional. Objetivo: Elaborar un plan estratégico que impulse el desarrollo local de las comunidades en situación de pobreza. Método: investigación cuantitativa, descriptiva y transversal desarrollada en una comunidad del sureste de México, con una población total de 500 familias. Se determinó una muestra representativa de 90 hogares, habiéndose aplicado un cuestionario estructurado a partir de investigaciones relacionadas. Resultados. Se identificaron los factores determinantes de pobreza y marginación en la localidad, y se establecieron las bases para la elaboración de un plan estratégico para el desarrollo local.

Palabras clave: planeación estratégica, desarrollo regional, desarrollo local, pobreza, plan estratégico

\footnotetext{
a Universidad Juárez Autónoma de Tabasco. UJAT correo: jfgr55@hotmail.com

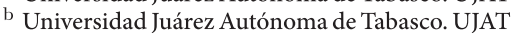

${ }^{c}$ Universidad Tecnológica del Estado de Tabasco. UTTAB

d Universidad Juárez Autónoma de Tabasco. UJAT

${ }^{\mathrm{e}}$ Universidad Intercultural del Estado de Tabasco. UIET

* Autor de correspondencia
} 


\begin{abstract}
Strategic planning represents a viable alternative to promote local and regional development. Objective: To prepare a strategic plan that promotes the local development of communities in situations of poverty. Method: quantitative, descriptive and cross-sectional research developed in a community in the southeast of Mexico, with a total population of 500 families. A representative sample of 90 households was determined, having applied a structured questionnaire based on related research. Results. The determining factors of poverty and marginalization in the locality were identified, and the bases were established for the elaboration of a strategic plan for local development.
\end{abstract}

Keywords: strategic planning, regional development, local development, poverty, strategic plan.

\title{
Introducción
}

La pobreza, marginación y desigualdad que afecta en gran medida a la población mexicana constituyen problemas socioeconómicos de naturaleza estructural, compleja y multidimensional. Dicha problemática está presente en el ámbito urbano y rural; sin embargo, son los habitantes de las comunidades y pueblos rurales quienes sufren más sus consecuencias adversas: ingresos precarios y falta de empleo, carencias sociales, rezago educativo y en salud, inseguridad alimentaria, desnutrición, inseguridad, deficiente infraestructura y vías de comunicación, etc., indicadores característicos del subdesarrollo y rezago social prevaleciente que los obliga a migrar de sus lugares de origen. Ello a pesar del derecho fundamental a una vida digna. Ante ello, es responsabilidad del Estado nacional asegurar el cumplimiento de dicho derecho, creando leyes, políticas y programas que impulsen el bienestar de la población, especialmente de los segmentos sociales más vulnerables.

Constitucionalmente, el Estado mexicano es responsable de la planeación económica nacional que impulse el desarrollo y bienestar de la población. En materia de desarrollo regional mucho se ha avanzado en México, sobre todo en el norte y centro del país, donde algunas entidades federativas observan un alto grado de desarrollo económico e industrial, así como elevadas condiciones de vida de sus habitantes, sobre todo en sus áreas urbanas. En contraste, en el sur-sureste mexicano prevalece el rezago económico e industrial, así como elevados niveles de pobreza, marginación y desempleo. Ante ello, es necesario impulsar el desarrollo homogéneo del país, así como elevar el nivel de bienestar económico y social de toda la población, disminuyendo los niveles de pobreza y desigualdad, rezago e inseguridad presentes en buena parte del país (Miguel (2004).

En la búsqueda de este propósito, el Estado cuenta con una importante herramienta metodológica de apoyo como lo es la planeación estratégica, la cual instrumentada a través de un plan estratégico efectivo podría contribuir al impulso del desarrollo local de las comunidades y pueblos en situación de pobreza. Dicho plan, cumpliéndose ciertas condiciones, podría ser replicado a otras localidades de características similares, de ahí su importancia metodológica. Por ello, el objetivo general de esta investigación es proponer un plan estratégico que impulse el desarrollo local de las comunidades en situación de pobreza, Dicho plan contiene los principales elementos metodológicos de la planeación estratégica. 
La investigación es relevante, toda vez que aporta una propuesta metodológica para el desarrollo local de las comunidades en situación de pobreza y alta marginación. El Plan Estratégico de Desarrollo Local propuesto podría constituir un instrumento de trabajo para la planificación del desarrollo local en los tres niveles de gobierno. Además, constituiría una importante herramienta metodológica al alcance de las comunidades rurales mediante la cual podrán involucrarse en su propio proceso de cambio, identificando sus potencialidades, fortalezas y debilidades, así como las organizaciones a constituir y las inversiones a realizar para encausar el desarrollo local, haciéndolos de esta manera sujetos de su propio desarrollo.

\section{Conceptualización del desarrollo}

En la teoría económica, el concepto de desarrollo se asocia al de crecimiento económico, asumiéndose por los primeros teóricos de la economía que la mejora de una sociedad se daría en la medida en que se incrementaran las inversiones y la productividad, lo que derivaría en un aumento de los ingresos de las familias, y por consiguiente, en la mejora de sus condiciones de vida. De esta manera, el Producto Interno Bruto (PIB) y el PIB per cápita fueron los indicadores macroeconómicos por excelencia (Boisier 2001). Sin embargo, surge de inmediato la pregunta obligada: ¿cuánta congruencia habrá entre el PIB per cápita y las condiciones reales de bienestar de las familias? Evidentemente, la debilidad de este indicador es que no mide la brecha de desigualdades sociales. Así, en la conceptualización del desarrollo debe estar implícita la mejora de las condiciones de vida de la sociedad, más allá del incremento de los ingresos. Por lo tanto, el desarrollo no es un hecho terminado en el tiempo, sino más bien un proceso en el que los objetivos y las acciones están encaminadas a garantizar el bienestar de la población de manera creciente y constante (Miguel, 2004). No obstante, el concepto cobra fuerza durante la posguerra, con la creación de diversos programas de apoyo para el desarrollo de los países pobres, cuya organización y supervisión recayó en el Banco Mundial (BM) y el Fondo Monetario Internacional (FMI) (Miguel, 2004).

No obstante, el desarrollo económico debe asumirse como un proceso a través del cual una nación logra alcanzar mejores estándares de vida de su población (Brue \& Grant, 2009). En este contexto, la ONU (2012) ha planteado diversas prioridades para el logro de un desarrollo sostenible que fomente la prosperidad, las oportunidades económicas, el bienestar social y la protección del medio ambiente; todo ello con la intención de mejorar las condiciones de vida de la población en general. De esta manera, el desarrollo económico busca un equilibrio en sus tres pilares básicos: económico, social y ecológico. Aquí, la pregunta central sería cómo lograr el desarrollo económico que impulse el bienestar de la sociedad, el crecimiento económico sostenido y el cuidado del medio ambiente. Los economistas coinciden en cuatro elementos indispensables para el desarrollo: recursos humanos, recursos naturales, capital e innovación y cambio tecnológico (Samuelson \& Nordhaus, 2010). Si bien es cierto que el propósito central del desarrollo económico es mejorar los niveles de vida de las personas, no debe dejarse de lado que no hay desarrollo sin crecimiento económico; es decir, el crecimiento económico está implícito en el desarrollo (Samuelson \& Nordhaus, 2010).

Así, aunque la teoría del desarrollo económico sostiene que el PIB per cápita no es un indicador objetivo para medir el bienestar de la población, Krugman y Wells (2014) explican que éste se usa como medida resumida del progreso del país a lo largo del tiempo, y que el crecimiento económico a largo plazo depende casi totalmente de la variable productividad, la cual obedece el aumento del capital físico, el aumento del capital humano y el progreso tecnológico. En síntesis, no hay desarrollo económico sin crecimiento económico, pero el crecimiento en sí mismo no genera desarrollo económico. Esto justifica la intervención del Estado en la actividad 
económica a efectos de corregir las fallas del mercado, procurando una distribución justa y equitativa de la riqueza (Espinoza, 2008). No obstante, la historia económica demuestra que el crecimiento económico ha generado una gran brecha de desigualdad, misma que se traduce en marginación y pobreza. Esto se debe a que el retorno del capital es más alto que la renta del trabajo. Si bien el crecimiento económico no resuelve esta brecha social, no deja de ser un elemento fundamental del desarrollo económico (Piketty, 2014).

Tradicionalmente el estudio del crecimiento económico se ha abordado desde dos grandes enfoques: el crecimiento económico hacia afuera y el crecimiento económico hacia adentro. En el primero, predomina la apertura al libre mercado; el Estado deja de intervenir como regulador de la economía y solo se dedica administrar justicia, y las leyes del mercado se encargan de la asignación de los recursos. En el segundo enfoque, el Estado interviene aplicando regulaciones para proteger el mercado interno e incentivar a las empresas nacionales, procurando la sostenibilidad de las mismas con el paso del tiempo. En ambos enfoques de crecimiento, se esperan alcanzar las rentas nacionales que permitan el bienestar de la población (Huerta \& Chávez, 2003). El enfoque de desarrollo regional se encuentra en la categoría del modelo de crecimiento hacia adentro, en el que las inversiones están dirigidas a desarrollar los sectores nacionales, considerando las vocaciones territoriales y los agentes económicos que lo propician.

Así, el desarrollo regional consiste en un proceso de cambio estructural localizado espacialmente, asociado a un proceso permanente de progreso de la propia región, así como de sus habitantes colectivamente e individualmente. Es decir, el desarrollo regional debe combinar tres dimensiones: la dimensión espacial, la dimensión social y la dimensión individual. Se trata de un proceso en el que la región es un sujeto colectivo (Boisier, 2001). El concepto de desarrollo regional se ha considerado en dos vertientes: subordinado al contexto nacional o independiente del mismo. En el primer caso, el desarrollo regional se ha entendido como un proceso de desarrollo nacional a escala regional, considerándose las características económicas, sociales y físicas del cambio en una zona durante un periodo de tiempo; en el segundo, el desarrollo regional se concibe como un aumento del bienestar en la región expresado en indicadores tales como el ingreso per cápita, su distribución entre los habitantes, el acceso a los servicios sociales y la adecuación de normas legales y administrativas. Aunque algunos prefieren ver el desarrollo regional como un proceso dependiente del desarrollo nacional, en muchas regiones pobres se han superado aspectos desfavorables o se han creado nuevas situaciones favorables que mejoran la calidad de vida de sus habitantes gracias a la planificación del desarrollo regional (Miguel , 2004).

El concepto de desarrollo endógeno nace como una respuesta al pensamiento dominante de los cincuenta y sesenta, el desarrollo territorial basado en el paradigma industrial de producción en serie y en la difusión de las innovaciones y los impulsos de cambio. Contrariamente, el desarrollo endógeno puede ser entendido como una propiedad emergente de un sistema territorial que posee un elevado stock de capitales intangibles y sinérgicos. Es decir, se produce como resultado de la integración de los actores locales y de varias formas de capital intangible, en el marco de un proyecto político colectivo del desarrollo del territorio (Boisier, 2001). Este autor plantea que la endogeneidad del desarrollo regional debe entenderse como un fenómeno que intercepta cuatro planos importantes que generan un escenario ocupado por una variedad de actores públicos y privados quienes interactúan en diversos niveles: 1. En el plano político, se identifica la creciente capacidad regional para tomar decisiones en torno a las diferentes estrategias de desarrollo y al uso de instrumentos correspondientes en esta materia, es decir, la capacidad de diseñar y ejecutar políticas de desarrollo, así como la capacidad de negociar. 2. En el plano económico, se tiene que considerar la apropiación y reinversión regional de parte del excedente para diversificar la economía y asegurar la sustentabilidad en el tiempo. En el plano científico y tecnológico, el territorio visto como un sistema debe tener la capacidad interna de generar sus propios 
impulsos tecnológicos de cambio o ser capaces de hacer modificaciones cualitativas en el sistema. 4. Finalmente, en el plano cultural, el desarrollo endógeno debe gestarse en el interior del territorio, considerando las características, conocimientos y creencias de su población

En síntesis, el desarrollo endógeno mira al interior del territorio en busca de las potencialidades que detonen el desarrollo de la comunidad e impulsen el bienestar a una escala mayor en el futuro. Dignificar la vida supone que existe un conjunto de necesidades insatisfechas colectivas que deben ser atendidas y un elemento clave es la construcción de una identidad colectiva que refleje el ser integral de la comunidad. El desarrollo a partir de los recursos locales demanda una vida digna y la construcción de identidad colectiva (Fuenmayor, 2003). En este sentido, el desarrollo endógeno se presenta como una idea nacionalista del desarrollo, el ejercicio de la soberanía territorial. Y es lógico desde el instante en que se espera propiciar el desarrollo desde el interior a partir de los recursos que se dispone. Este enfoque plantea interrogantes de tipo global, por ejemplo, ¿qué lugar ocupa el exterior en el desarrollo endógeno, tiene algún valor? Porque el mundo es cada vez más globalizado y los temas de desarrollo no deben limitarse a considerar esta complejidad. Por esto, el mundo se parece más a un mercado global, con rezagos en los países que aún no incursionan plenamente en esta dinámica (Pilonieta \& Ochoa, 2006).

Queda claro que los agentes del desarrollo endógeno son: autoridades locales y regionales, iniciativa privada y la sociedad con su conocimiento cultural e innovaciones tecnológicas, que conduce a la transformación social. Garafoli, citado por Boisier (2001), dice que el desarrollo endógeno significa la capacidad para transformar el sistema socioeconómico; la habilidad para reaccionar a los desafíos del exterior; la promoción del aprendizaje social; y la habilidad de introducir formas específicas de regulación social a nivel local que favorecen el desarrollo de las características anteriores. Y concluye, el desarrollo endógeno simplemente es la habilidad para innovar a nivel local. Entonces, este enfoque de desarrollo coloca las piezas del rompecabezas e indica cómo deben moverse para situar todo en su lugar. De manera que, el desarrollo local no puede concebirse sin desarrollo endógeno, sin embargo este concepto es más amplio, tanto que debe considerarse en los temas de desarrollo regionales (Boisier, 2001). El reto está delante, implica trabajo duro y perseverancia. Innovar a nivel local es clave para impulsar el bienestar de las comunidades en situación de pobreza.

En este contexto, el desarrollo local se asume como un proceso de cambio favorable de una sociedad a partir de su identidad y su propio territorio, durante el cual se generan y fortalecen sus dinámicas económicas, sociales y culturales, facilitando la articulación de cada uno de estos subsistemas y logrando mayor intervención y control entre ellos. (Casanova, citado por Alcañiz, 2008). De esta manera, el desarrollo local procura determinar dos aspectos: primero, cuál es el potencial de recursos con el que se cuenta; y segundo, cuáles son las necesidades que se requieren satisfacer tanto de las personas como de las comunidades, colectividades, municipios y la sociedad en general. El desarrollo local parte del análisis de los recursos disponibles con los que se impulsará el bienestar de una comunidad pequeña o grande, con base en un proceso de planeación estratégica (Silva \& Sandoval, 2012). Este enfoque surge como respuesta a las fuertes transformaciones producidas por la acumulación de capital, que plantea problemas de regulación como la gestión de trabajo o la adaptación y difusión de la tecnología moderna que las instituciones del pasado afrontaron, pero que ahora son más complejas, de manera que los instrumentos de intervención estatal han perdido eficacia en la regulación de la economía, por lo que estos cambios obligan al Estado a intervenir estratégicamente. Ante ello, la reestructuración del Estado está impulsando nuevas estrategias en la gestión pública, como lo es la política de desarrollo local. De esta manera, muchas comunidades, especialmente europeas, han intentado dinamizar el ajuste de los sistemas productivos locales (Boisier, 2001). 
Por su parte, el enfoque de Desarrollo Sustentable tiene sus orígenes en la década de los ochenta, cuando la Organización de las Naciones Unidas (ONU) creó la Comisión Sobre el Medio Ambiente y el Desarrollo y su famoso informe Nuestro Futuro Común, también conocido como El Informe Brundtland. En el mismo se señalaba con claridad que la sociedad debía modificar su estilo y hábitos de vida para evitar una crisis social y la degradación de la naturaleza. De esta manera, el desarrollo sustentable debía satisfacer las necesidades de la generación presente, sin comprometer la capacidad de las generaciones futuras para satisfacer sus propias necesidades (Ramírez, Sánchez, \& García, 2004). La definición anterior contempla dos conceptos importantes: 1) el concepto de necesidades, especialmente las necesidades básicas de las personas vulnerables, y 2) preservación del medio, es decir, reconoce que las capacidades para la satisfacción de necesidades provienen de la naturaleza, por lo tanto, es responsabilidad de la generación presente satisfacer sus necesidades, sin comprometer las capacidades de satisfacción de las generaciones futuras. En este contexto, en septiembre de 2015 se estableció la Agenda 2030 para el Desarrollo Sostenible, misma que contempla diecisiete objetivos para alcanzar el desarrollo sostenible, entre los que destaca el fin de la pobreza (ONU, 2015). Estos objetivos, como señala Zarta (2018) persiguen un proceso armonioso de desarrollo sustentable en el cual colaboren todas las disciplinas del conocimiento, especialmente en lo económico, social, ambiental, cultura, así como un sistema de valores correspondientes.

\section{Pobreza. Caracterización y situación actual}

Uno de los problemas más debatidos dentro del ámbito de la economía, la política, la filosofía y la ética es la pobreza, entendida como una condición socioeconómica que limita el bienestar de las personas y que constituye un asunto de naturaleza multidimensional y complejo (García, 2016). Al tratarse de un problema complejo, su estudio y abordaje requiere enfoques multidimensionales e interdisciplinarios para comprender sus causas y diseñar políticas adecuadas para enfrentarlo. La pobreza es un problema latente a nivel mundial. Según el Banco Mundial (2019), para el 2018 había 7 mil 594 millones de habitantes en el mundo, de los cuales 736 millones se encontraban en situación de pobreza extrema. Esto quiere decir que poco más del 10\% de la población mundial sobreviven diariamente con un ingreso por debajo de 1.90 dólares.

En México la pobreza es un tema preocupante. Así, en el año 2018 de una población total de casi 125 millones de habitantes, 52.4 millones, se encontraban en situación de pobreza multidimensional, lo que equivale al 41.9\% del total (CONEVAL, 2018). Esto implica que tal población tiene al menos una carencia social y su ingreso es insuficiente para adquirir los bienes y servicios que requiere para satisfacer sus necesidades alimentarias. Lo más alarmante es que el 7.4\% de la población total se encontraba en pobreza extrema; es decir, 9.3 millones de personas vivían con un ingreso tan bajo que ni aun gastándolo exclusivamente en alimentos podían adquirir los nutrientes necesarios para una vida sana, y además tenían tres o más carencias sociales. Parte del problema de la pobreza radica en la baja efectividad de las políticas públicas implementadas para enfrentarla. Así, En el periodo 2015 al 2017 operaron en el país 5,491 programas sociales de los cuales sólo 83 estaban dirigidos a combatir las carencias sociales y eran considerados prioritarios. Sin embargo, de acuerdo al CONEVAL estos programas no dieron los resultados esperados por deficiencias en su diseño e implementación (Roldán, 2017).

Geográficamente, la mayor parte de la población en situación de pobreza se concentra en las zonas rurales, lo que obedece a la exclusión social y económica a la que están expuestas, y que se traduce en limitaciones de acceso a los servicios de educación y salud, así como a los mercados laborales, limitados canales 
de comercialización de los productos locales, así como deficientes vías de comunicación. Ante ello es necesario promover la inclusión social y el desarrollo de capacidades locales (Portales, 2014). Para lograrlo, la planeación estratégica del desarrollo local se presenta como una alternativa efectiva para enfrentar la pobreza (Herrera, 2013). A partir del diseño de un plan estratégico de desarrollo local, el Estado en sus tres niveles de gobierno (federal, estatal y municipal) puede focalizar mejor las políticas y programas de apoyo a las comunidades, proveyéndoles mejores servicios básicos y mayores elementos para el desarrollo local sustentable.

\section{Planeación estratégica como instrumento de desarrollo local}

La planeación es la primera fase del proceso administrativo de cualquier organización, pública o privada. Para el Estado, la planeación es un instrumento de la política de desarrollo que hace posible establecer políticas efectivas de crecimiento, y facilita la toma de decisiones sobre bases objetivas y estratégicas (Chapoy, 2003). Según Chandler (2003), la estrategia consiste en el establecimiento de metas y objetivos a largo plazo de una organización, así como las acciones a emprender y los recursos necesarios para su consecución. Al respecto, Armijo (2011) comenta que la planeación estratégica es un ejercicio de formulación y establecimiento de objetivos prioritarios, cuya característica central es el establecimiento de los cursos de acción (estrategias) para alcanzarlos. La planeación estratégica está integrada por elementos consecutivos que dan orden a su elaboración: misión, visión, valores organizacionales, objetivos estratégicos, análisis interno y externo, estrategias, líneas de acción e indicadores de desempeño. Todos estos elementos se plasman en un documento rector denominado plan estratégico de desarrollo. En este contexto, la planeación del desarrollo local debe ser estratégica, a efectos de priorizar los objetivos que se propone alcanzar.

La planeación estratégica del desarrollo local exige la participación activa de los agentes involucrados, como son la población beneficiaria, autoridades locales, instituciones públicas y universidades, agencias de desarrollo local, organizaciones sociales, etc. (Silva \& Sandoval, 2012). En general, la planeación estratégica local implica realizar un diagnóstico de las fortalezas y debilidades presentes en la localidad, y a partir de ello elaborar un plan estratégico que contenga las acciones que deben emprenderse para enfrentar los problemas sociales y económicos complejos que enfrentan, como son entre otros la pobreza, desigualdad y marginación. En base a los referentes teórico-metodológicos antes expuestos, se determinó el siguiente algoritmo teórico para el desarrollo local, adaptado de la propuesta de Miguel (2004), mismo que en lo sucesivo orienta el diseño de la investigación y la propuesta planteada. 


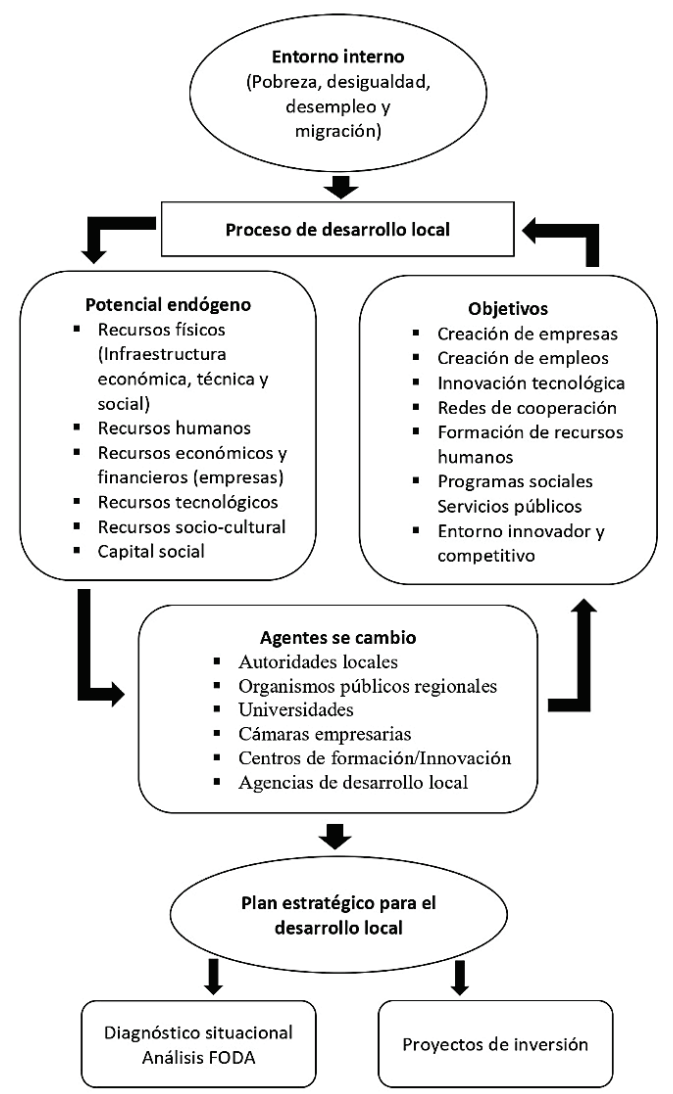

Fuente: (Adaptado de Miguel, 2004) 


\section{Método}

Enfoque. Investigación cuantitativa que parte de una idea que deriva en el planteamiento de un problema de investigación, preguntas y objetivos; se sustenta en una revisión de la literatura y se establece un marco teórico, así como una hipótesis a demostrar, en la cual se determinan las variables de estudio, mismas que a partir de la información captada en el trabajo de campo son medidas con estadística descriptiva (Hernández, Fernández y Baptista, 2014). Tipo de investigación. Investigación descriptiva y transversal, cuyo propósito es describir y analizar las variables identificadas, así como la interrelación existente entre ellas (Hernández, Fernández, \& Baptista, 2014). Diseño. Como caso de estudio se tomó una localidad en situación de pobreza y marginación del sureste de México en la que viven 500 habitantes. El tamaño de muestra es de 90 hogares, y se determinó mediante muestreo aleatorio simple, considerándose un intervalo de confianza del 95\%, y un margen de error del 5\%. La técnica para recolección de la información fue diseñada a partir de los objetivos de la investigación, habiéndose aplicado a los sujetos de estudio un cuestionario con preguntas cerradas, construido en base a investigaciones precedentes con características similares, así como en el cuestionario utilizado en el censo de ingreso-gasto aplicado por el INEGI en 2018. De igual manera, se tomó como referencia el manual de Metodología para la elaboración de estrategias de desarrollo local de Silva y Sandoval, publicado por la CEPAL en el año 2012. Para el análisis cuantitativo de la investigación se aplicó estadística descriptiva.

\section{Resultados}

Derivado del análisis efectuado a la información recolectada en el trabajo de campo, se deducen entre otros los siguientes resultados: 1. Al igual que en el resto de las comunidades en situación de pobreza del país, las características sociodemográficas de la localidad estudiada se corresponden con la transición demográfica presente en los países latinoamericanos, como son la presencia mayoritaria de población joven y adulta, bajo nivel de escolaridad, precariedad del empleo y por lo tanto del nivel de vida de sus habitantes. 2. Debido a las condiciones de marginación y bajo nivel de escolaridad, el trabajo desarrollado por la población es precario y de bajo nivel agregado. De esta manera, las ocupaciones de la población masculina entrevistada están centradas en las actividades propias del campo y en la generación de productos del sector primario con bajo valor agregado, los cuales se comercializan en la comunidad y en pocos casos tienen alcance externo a la misma.

3. También se registran actividades de ganadería y pesca a baja escala. El sector servicios comienza a cobrar importancia a través del comercio y los servicios técnicos de carpintería, plomería, electricistas, mecánicos y veterinarios. En el caso de las mujeres, éstas se dedican generalmente a labores del hogar, actividad que en la mayoría de los casos no implica remuneración alguna. Asimismo, existe un alto nivel de desocupación en ambos sexos. 4. Dada la precariedad de sus actividades económicas y su baja escolaridad, es natural que los ingresos de la población sean insuficientes para satisfacer sus necesidades básicas, no solo alimenticias sino también de educación, salud y vivienda. Así, se encontró que el 52\% de los entrevistados percibe menos de 500 pesos mexicanos semanales por su trabajo principal, el cual distribuido en un hogar con un promedio de 5 personas obliga a una vida precaria y limitada, pues dicho ingreso se usa principalmente para gastos de alimentación (65\%), educación (20\%), salud y medicamentos (8\%) y vestimenta (5\%). Asimismo, en el 60\% de las familias encuestadas la figura paterna aporta el ingreso total del hogar, mientras que el $40 \%$ restante es aportado por diferentes miembros de la familia. Por las actividades extras que realizan y los apoyos gubernamentales que perciben las familias, el ingreso familiar semanal se incrementa. 
5. Los apoyos gubernamentales de transferencias monetarias han incrementado notablemente el ingreso familiar de los habitantes en situación de pobreza, lo que ha coadyuvado a reducir las carencias alimentarias. El impacto de los programas de apoyo que están beneficiando a los habitantes de la comunidad estudiada es relevante. Así, el Programa Sembrando Vida apoya al 32\% de las familias; Becas de Educación Básica un 40\%; Becas de Educación Media Superior 19\%; Becas de Educación Superior 6\%; Pensión para Adultos Mayores 13\%; Jóvenes Construyendo el Futuro 10\% y Personas con Discapacidad 3\%. 6. Un signo distintivo de la pobreza y marginación de la población es la falta de acceso a diversos bienes públicos básicos. Las carencias sociales se refieren a la privación del acceso a capacidades básicas para la vida y el bienestar como son los servicios básicos de educación, salud y vivienda. Así, en la comunidad estudiada la mitad de la población apenas si cuenta con estudios de nivel primaria, el 30\% de secundaria, el 12\% de preparatoria, y solo el 2\% estudió alguna profesión. Así mismo, el 6\% es analfabeta, pues no sabe leer ni escribir.

7. Respecto a la disponibilidad y acceso otros servicios básicos para el bienestar y el desarrollo local, el 90\% de la población dispone de los servicios de energía eléctrica y agua potable, aunque su suministro es deficiente e irregular. Ambos servicios están relacionados: si no hay energía eléctrica, el pozo de distribución de agua potable no funciona. Al momento que se aplicó la encuesta, la comunidad tenía 20 días sin agua potable debido a fallas en la bomba de distribución. Por otra parte, el 91\% de la población entrevistada coincidió en que los caminos y carreteras que comunican a la localidad se encuentran en mal estado y cada vez se deterioran más, sin que ninguna autoridad atienda este problema. Por lo mismo, el transporte público es deficiente e insuficiente para satisfacer las demandas de traslado de los habitantes de la comunidad.

8. Un aspecto relevante para el desarrollo local es la percepción que tiene la sociedad acerca de su propia realidad y la manera de superar sus problemas. Con tal propósito, el trabajo de campo incluyó un apartado especial. Al respecto, todos los entrevistados consideraron que la comunidad cuenta con importantes recurso naturales susceptibles de aprovechamiento, como son amplias extensiones de tierras fértiles para la agricultura y abundantes manglares y lagunas para la pesca y ostricultura, así como la elaboración de artesanías y la carpintería. Otro aspecto potencial de desarrollo sería el emprendimiento del turismo ecológico sustentado en la riqueza natural que rodea a la comunidad. Al respecto, debe destacarse la satisfacción de la población con los programas públicos implementados por la administración federal actual. Así, la tierra fértil está siendo aprovechada para la reforestación a través del programa sembrado vida, del cual el 32\% de la población está resultando beneficiada, ya sea porque reciben un ingreso en su calidad de propietarios, o porque cuentan con un empleo y reciben un ingreso como trabajadores agrícolas campesinos.

\section{Discusión}

La situación de pobreza y rezago social en México constituye un problema estructural complejo. En el año 2018, el $41.9 \%$ de la población total se encontraba en pobreza multidimensional, y lo que es más grave, el 7.4\% en pobreza extrema, sobre todo en las regiones rurales del sureste de México. Asimismo, en materia de pobreza extrema los indicadores señalan que Tabasco se encuentra por encima de la media nacional, siendo uno de los estados más vulnerables en materia de pobreza y rezago social a nivel nacional. De ahí el interés por impulsar estrategias para el desarrollo local, a efectos de mejorar las condiciones de vida de los habitantes de la áreas rurales, a partir del aprovechamiento estratégico de las potencialidades que estos territorios poseen. En la 
comunidad estudiada se encontró que las familias rurales perciben ingresos insuficientes para satisfacer sus necesidades básicas, motivo por el cual permanecen en situación de pobreza extrema, y son a la vez las que más privaciones sociales presentan, lo que les limita su derecho a una vida digna. Manifiestan también un importante rezago en materia de educación, salud y seguridad social, así como múltiples carencias en la calidad de su vivienda, limitaciones en el acceso a la energía eléctrica, servicios de agua potable, carreteras y transporte público.

Por otro lado, los habitantes de la comunidad cuentan con una perspectiva de desarrollo local e identifican claramente los recursos potenciales disponibles y su posible aprovechamiento, lo que facilita la implementación de un proceso de planeación estratégica. Así, identificada la percepción de la comunidad respecto a su circunstancia actual, así como las principales debilidades, fortalezas y potencialidades para el desarrollo local, resulta pertinente la elaboración de un plan estratégico de acción que debidamente aplicado posibilite en el mediano y largo plazo el desarrollo local, contando con la participación activa de la propia población y sus autoridades, el Estado en todos sus niveles y las organizaciones públicas y privadas que deban concurrir.

En este contexto, el desarrollo local representa una alternativa viable para impulsar la superación de la situación de pobreza y marginación presente en muchas regiones del país, de ahí la importancia de impulsar procesos de planeación estratégica para el desarrollo integral por parte del estado nacional. Lograr que la planeación estratégica sea un instrumento efectivo mediante el cual las comunidades pobres y marginadas participen activamente en el aprovechamiento racional de los recursos naturales disponibles en su localidad constituye un propósito esencial. Ello presupone un adecuado acompañamiento organizativo y capacitación técnica efectiva para el mejor aprovechamiento de los recursos productivos disponibles, así como la mejora de los procesos de producción tradicionales (Herrera, 2013).

De esta manera, la planeación estratégica del desarrollo local constituye un instrumento preponderante en las iniciativas de combate a la pobreza. Se trata de una acepción al desarrollo que busca posicionarse como la más adecuada en la creación de procesos en la reducción de los índices de marginación y pobreza en que se encuentran las comunidades rurales del país. Se basa en la capacidad de desarrollar su potencial por medio de la generación de acciones locales, que a su vez, se integran en el entorno global. Busca atender las problemáticas relacionadas con la creación de medios y capacidades que mejoren la calidad de vida, a través de la participación social y el aprovechamiento de los recursos que se encuentran en un territorio determinado (Portales, 2014).

\section{Propuesta}

La planeación estratégica del desarrollo local significa una alternativa viable para la superación de la pobreza y el rezago social y económico de las comunidades marginadas del desarrollo nacional y regional. Por ello, se concluye con la elaboración de una propuesta de plan estratégico para impulsar el desarrollo local de la comunidad analizada. Puesto que las características de la misma son similares a las comunidades en situación de pobreza de México y otras latitudes, dicha propuesta constituye una propuesta metodológica para el desarrollo local aplicable en el ámbito nacional e internacional. Dado que el desarrollo local y la superación de la pobreza constituyen procesos dinámicos de mediano y largo plazo, la presente propuesta de plan estratégico aplicado debe ser adaptado a las características específicas de las comunidades y regiones durante la fase de implementación. 


\section{Propuesta de plan estratégico para el desarrollo de comunidades en situación de pobreza y marginación}

El plan estratégico para impulsar el desarrollo local de las comunidades en situación de pobreza y marginación que a continuación se detalla representa una propuesta ciudadana orientada a mejorar las condiciones de vida de la población, y se sustenta en el aprovechamiento racional y estratégico de las potencialidades que los mismos territorios poseen.

A partir del presente plan estratégico de desarrollo local, el gobierno en sus diversas instancias, las organizaciones civiles y las organizaciones no gubernamentales pueden focalizar de manera efectiva las políticas y programas de apoyo a las comunidades seleccionadas, proveyéndoles de mejores servicios públicos e infraestructura de carreteras, luz eléctrica, agua potable, drenaje, servicios de salud, escuelas, vivienda, empleo, etc. En fin, mediante esta estrategia de planeación para el desarrollo local se vislumbra un futuro esperanzador para las comunidades y sus habitantes que enfrentan los estragos generados por la pobreza y marginación.

La propuesta de plan estratégico tiene como propósito impulsar el desarrollo local y coadyuvar a reducir las condiciones de pobreza y marginación de sus habitantes. Metodológicamente está estructurado en los siguientes apartados: 1) Misión; 2) Visión; 3) Valores; 4) Objetivo general; 5) Análisis FODA; 6) Objetivos estratégicos, estrategias y líneas de acción, y 7) Programas y proyectos prioritarios. Dado que el desarrollo local constituye un proceso dinámico de cambio, el plan no constituye una propuesta acabada y por lo tanto, debe adecuarse constantemente a las condiciones específicas de cada región y/o localidad en la cual pretenda ser aplicado.

\section{Misión}

Somos una comunidad gestora de un desarrollo local participativo, integral y sostenible, que propicie bienestar para todos los habitantes, a partir del aprovechamiento de la vocación territorial y de esta manera, reducir los niveles de pobreza.

\section{Visión}

La comunidad será identificada por sus niveles de integración, participación, inclusión y capital social empleados eficazmente en la gestión pública de infraestructura y bienes de capital que impulsen el desarrollo local reduciendo las condiciones de pobreza de las familias.

\section{Valores y principios}

\section{Dignificación del ser humano}

El ser humano es el tema central del desarrollo económico y este no tiene sentido si no dignifica a los individuos. La meta del desarrollo local siempre será mejorar las condiciones de vida de las personas permitiéndoles ejercer sus derechos individuales, sociales y económicos.

\section{Inclusión}

El bienestar es tarea de todos y para todos. Es la capacidad de involucrar a todas las personas dentro de los procesos de planeación que se desarrollan en el territorio desde el reconocimiento de las diferencias. 


\section{Trabajo comunitario}

Velar porque todos los habitantes participen activamente en las decisiones y acciones necesarias para el desarrollo local, hechos con sentido de responsabilidad, solidaridad y progreso.

\section{Identidad territorial}

Capacidad que tiene la comunidad de reconocerse a sí misma como un territorio con personalidad jurídica que le permite planear, gestionar y desarrollar los programas y proyectos, articulada con diferentes instancias, públicas y privadas, sin perder su identidad.

\section{Sustentabilidad}

El desarrollo local debe ser amigable con la naturaleza. Es necesaria una administración eficiente y racional de los recursos naturales que permita mejorar el bienestar de la población actual sin comprometer la calidad de vida de las generaciones futuras. Para ello las acciones deben dirigirse a garantizar el buen estado ambiental, la gestión del riesgo y la recuperación de ecosistemas.

\section{Transparencia}

El desarrollo de los programas y proyectos deben estar fundamentados en acciones claras, honestas, con compromiso y servicio a la comunidad. El éxito del plan requiere que las cuentas sean rendidas en tiempo y forma.

\section{Equidad}

Se requiere determinar el impacto social de los programas y proyectos y priorizar los grupos que serán beneficiados de acuerdo con el reconocimiento de su condición específica.

\section{Objetivo general}

Reducir los niveles de pobreza en la localidad a través de la gestión de programas y proyectos dirigidos a impulsar el desarrollo local, integral, participativo y sustentable, que dignifique a la persona humana, proveyéndoles de los activos y capacidades para el ejercicio de sus derechos; de manera que, la comunidad se constituya en un marco de referencia a nivel municipal y estatal por su integración, participación y capital social utilizados eficazmente en la promoción de su bienestar.

\section{Análisis FODA}

En un ejercicio de diálogo social con los habitantes de la comunidad y sus autoridades, se lograron identificar las principales fortaleza y debilidades prevalecientes en la localidad, así como las oportunidades y amenazas presentes en su entorno. 


\section{Objetivos estratégicos, estrategias y líneas de acción}

1er. Objetivo estratégico: Potenciar el capital social que propicie la integración en la comunidad a través de la cooperación, confianza, reciprocidad y solidaridad entre sus miembros, con fines productivos y de gestión pública y privada, que en el largo plazo favorezcan el desarrollo local.

Estrategias:

1. Fortalecer el sentido de identidad en la comunidad.

2. Gestionar recursos para crear infraestructura que permita desarrollar actividades deportivas y recreativas.

3. Habilitar espacios que permitan la interacción social.

4. Organizar eventos que permitan fortalecer las relaciones entre los habitantes.

5. Integrar grupos para el desarrollo de habilidades y la incubación de proyectos de inversión.

6. Gestionar apoyos económicos grupales y familiares.

Líneas de acción:

1. Realizar campañas de integración social e identidad territorial.

2. Integrar equipos deportivos masculinos y femeninos, de adultos y jóvenes, y organizar torneos entre las comunidades vecinas.

3. Capacitar a grupos o familias en la gestión de créditos para proyectos emprendedores.

4. Monitorear los grupos y asociaciones formadas e impulsarlas a través de incentivos a la producción y permanencia.

2do. Objetivo estratégico: Fortalecer las capacidades cognitivas de los habitantes en los diferentes niveles, para que desarrollen una visión más aplica de la realidad, sean propositivos en el análisis de sus propios problemas y se reduzca el rezago educativo de la población.

Estrategias:

1. Desarrollar hábitos de lectura que permitan a la población analizar y reflexionar sobre los problemas del medio y las posibles soluciones.

2. Acercar el conocimiento científico y social a las personas de la comunidad a través del contacto con profesionistas del lugar: maestros, médicos, zootecnistas, juristas, ingenieros, etc. que les permitan abordar problemas de la vida diaria con un enfoque más amplio.

3.Gestionar incentivos económicos para quienes participen en actividades de formación educativas desarrolladas en la comunidad.

4. Fomentar servicios estudiantiles a la comunidad: alfabetización a adultos mayores, clases de regularización, planes de negocios, etc.

5. Reducir la deserción escolar.

6. Gestionar la instalación de servicios de internet gratuito en la biblioteca pública. 
Líneas de acción:

1. Habilitar espacios que permitan el contacto con literatura relevante y que promuevan la reflexión.

2. Habilitar la biblioteca como centro de lectura, investigación y reforzamiento de aprendizaje para los tres niveles educativos básicos.

3. En colaboración con el Delegado Municipal, la Directiva de Padres de Familia y la Dirección de las instituciones educativas acondicionar las instalaciones de las escuelas primarias, telesecundaria y preparatoria, de manera que por las tardes o los fines de semana se puedan utilizar para impartir conferencias, talleres y capacitaciones sobre diversos temas relacionados con el desarrollo.

4. Impartir conferencias, capacitaciones y talleres que fortalezcan los conocimientos de los niños, jóvenes y adultos, y contribuyan en su desarrollo.

5. Crear centros de regularización escolar en colaboración con los jóvenes de nivel medio superior y superior, apoyados con el programa federal Jóvenes Construyendo el Futuro.

6. Vigilar que los programas de becas y apoyos estudiantiles federales y estatales sean aplicados con eficiencia en la localidad.

7. Impulsar el acompañamiento de los padres hacia los hijos y de los maestros a hacia los estudiantes en su formación estudiantil para evitar la deserción escolar.

8. Impartir pláticas de orientación educativa y psicológica para padres e hijos.

3er. Objetivo estratégico: Gestionar el equipamiento del centro de salud comunitario de modo que sea suficiente y eficiente en brindar servicios de salud a los habitantes, garantizándoles este derecho indispensable para el desarrollo humano.

Estrategias:

1.Concientizar a la población sobre la importancia del control médico en la prevención de enfermedades.

2.Gestionar ante las instituciones municipales y estatales la ampliación del servicio de salud comunitario: infraestructura, servicio médico y medicamentos.

3. Ampliar los horarios de atención médica.

4. Garantizar la atención médica a mujeres embarazadas, personas de la tercera edad, niños lactantes y personas con capacidades diferentes.

5. Vigilar que los servicios de salud sean gratuitos y sin discriminación.

6. Vincular las becas y apoyos estudiantiles con la atención médica familiar.

Líneas de acción:

1.Realizar campañas de concientización, sobre la importancia del control médico en la prevención de enfermedades, en las escuelas y puntos de reunión.

2. Fortalecer las campañas de vacunación y de prevención de embarazos tempranos o no deseados.

3. Procurar un acercamiento con las instituciones de salud a nivel municipal y estatal para solicitarles oportunamente apoyos en esta materia.

4. Habilitar un espacio de alojamiento para los médicos de la comunidad. 
5. Crear una comisión de salud en la comunidad que procure el cuidado de los muebles e inmuebles del Centro de Salud, y además vigile que los servicios de salud sean provistos a todos los habitantes sin discriminación.

6. Monitorear que los beneficiarios de becas y apoyos estudiantiles tengan un control médico actualizado.

7. Que la comunidad opere como guarda principal de los bienes y servicios del Centro de Salud.

4to. Objetivo estratégico: Tramitar ante las instituciones municipales y estatales correspondientes la provisión de servicios públicos de calidad, que mejoren las condiciones de vida de los habitantes de la comunidad e impulsen la inversión de la iniciativa privada.

Estrategias:

1.Gestionar la pavimentación de las carreteras que permitan el libre tránsito automovilístico y peatonal.

2.Velar por el funcionamiento eficiente de la red eléctrica, el alumbrado público y la red de agua potable.

3. Ampliar la red de agua potable con la intención de que este servicio esté al alcance de todos.

4. Procurar vigilancia policiaca continua que brinde seguridad a la población y a la iniciativa privada resguardando el derecho de propiedad.

5. Fomentar la participación ciudadana en relación al tema de la inseguridad.

6. Impulsar una red de transporte público que satisfaga plenamente a los usuarios.

7. Gestionar programas de mejora de viviendas.

8. Gestionar ante la iniciativa privada la creación de redes de comunicación telefónica e internet.

9. Crear alternativas que posibiliten la seguridad social de los habitantes de la comunidad.

Líneas de acción:

1. A través de las autoridades y asociaciones locales, gestionar ante las instituciones municipales y estatales la pavimentación de las carreteras, que en el corto plazo permitan el contacto con la comunidad.

2. Gestionar ante las autoridades competentes la mejora de los servicios de luz eléctrica, alumbrado público y la ampliación de la red eléctrica para que estos servicios se brinde de manera segura y sin interrupciones.

3. En colaboración con las comunidades vecinas y las autoridades locales procurar la vigilancia policiaca continua para castigar el delito y resguardar el derecho a la propiedad privada.

4. Mejorar los servicios de transporte público a través de la gestión oportuna de este servicio, las demandas de las deficiencias presentadas y el impulso de la competencia entre los transportistas.

5. Investigar programas de creación y mejora de viviendas para las familiar más vulnerables de la comunidad.

6. En coordinación con la iniciativa privada impulsar la creación de redes de telecomunicación e internet, que permitan a la población la disposición de servicios.

7. En colaboración con las autoridades locales, municipales, estatales y federales, así como la iniciativa privada, gestionar la creación de programas accesibles orientados a la seguridad social considerando elementos básicos como el fondo para el retiro, pago por incapacidades laborales y seguros de decesos, a precios accesibles y con subsidios gubernamentales. 
5to. Objetivo estratégico: Impulsar el desarrollo local que posibilite los medios para la adquisición de las capacidades y activos necesarios, que propicien bienestar en las familias, a través del aprovechamiento óptimo de los recursos potenciales en la localidad, con programas basados en la sustentabilidad.

Estrategias:

1. Fomentar las actividades agrícolas, específicamente las relacionadas con la producción cacaotera y cocotera. Y frutos de temporada como el maíz y el frijol.

2. Apoyar las actividades ganaderas orientadas a la producción de carne y leche.

3. Impulsar las actividades pesqueras y de acuacultura generadora de empleo, ingresos y alimentación sana para la población.

4. Generar precios de garantía a los productos del sector primario que permitan la recuperación de costos, no se impongan a la ley de la oferta y la demanda y permitan ganancias justas a los productores.

5. Gestionar apoyos a proyectos grupales y familiares relacionados con la crianza y comercialización de pollos, cerdos y productos frutales. Del mismo modo apoyar a los comerciantes y proveedores de servicios técnicos.

6. En colaboración con las comunidades vecinas, las autoridades correspondientes y la iniciativa privada, diseñar y promover un proyecto de turismo alternativo relacionado con la preservación de los manglares y la fauna relacionada, en el que se pueda delinear recorridos a turistas con accesibilidad a servicios de hospedaje y alimentos de la región. Mismo que genere empleos a los habitantes de la comunidad y preserve en buen estado el ecosistema.

7.Capacitar a los habitantes de la comunidad en alternativas de reciclaje y procesamiento de deshechos.

Líneas de acción:

1. Gestionar programas y proyectos ante las autoridades municipales, estatales y federales de apoyo a las actividades agrícolas, ganaderas y pesqueras.

2. En las instancias correspondientes amparar los precios de garantía a los productos del sector primario.

3. Integrar grupos y asociaciones entre los habitantes de la comunidad con la finalidad de gestionar apoyos a actividades productivas y crear nuevos negocios.

4. Acudir a las instancias correspondientes con la intención de recibir de ellos asesoría técnica y apoyo de gestión en el diseño y promoción de un proyecto de desarrollo turístico alternativo en las comunidades colindantes.

5. Acudir a la iniciativa privada y presentarles el proyecto de desarrollo turístico alternativo con la intención de motivarlos a invertir en la comunidad y generar fuentes de empleo.

6. Gestionar apoyos relacionados con el procesamiento de basura y deshechos. 


\section{Programas y proyectos prioritarios}

Los programas y proyectos prioritarios en la gestión del desarrollo local de la comunidad, estarán dados de la siguiente manera:

Programa 1: Restauración y pavimentación de carreteras.

Programa 2: Fortalecimiento de las redes eléctrica y de agua potable, en colaboración con las instituciones responsables.

Programa 3: Capacitación y desarrollo de competencias para el trabajo productivo e innovador en el ámbito de la localidad, con el apoyo y acompañamiento de las instancias públicas corresponsables.

Programa 4. Acceso pleno y gratuito a los servicios de salud de calidad, tanto en atención primaria de la salud como en atención médica especializada.

Programa 5: Impulso a la autoconstrucción de vivienda digna para la población carente de la misma, en colaboración con las instancias federales, estatales y municipales involucradas.

Proyecto 1: Integración de grupos productivos para el fortalecimiento del capital social en la comunidad.

Proyecto 2: Impulso a la agricultura sustentable.

Proyecto 3: Impulso a las actividades ganaderas.

Proyecto 4: Impulso a las actividades pesqueras.

Proyecto 5: Construcción de una red de turismo alternativo para la conservación y contacto con los manglares y la fauna.

\section{Referencias}

Alcañiz, M. (2008): El desarrollo local en el contexto de la globalización. Revista de ciencias sociales Convergencia, vol. 15, núm. 47, mayo-agosto, 2008, pp. 285-315.

Armijo, M. (2011): Manual de planificación estratégica e indicadores de desempeño en el sector público, ILPES/CEPAL, Chile.

Banco Mundial. (2019): Pobreza. Panorma Mundial. Disponible en https://www.bancomundial.org/es/topic/ poverty/overview

Boisier, S. (2001): Desarrollo (local): ¿De qué estamos hablando? Transformaciones globales, Institucionales y Políticas de desarrollo local. Homo Sapiens, Rosario.

Brue, S., \& Grant, R. (2009): Historia del pensamiento económico. CENGAGE Learnning, México.

Chandler, A. (2003): Strategy and Structure. Chapters in the history of the American Industrial Enterprise. Beard Books, New York.

Chapoy, D. B. (2003): Planeación, programación y presupuestación. UNAM, México.

Coneval. (2018): ¿Qué es la medición de la pobreza? Disponible en https://www.coneval.org.mx/Medicion/MP/ Paginas/Que-es-la-medicion-multidimensional-de-la-pobreza.aspx

Fuenmayor, R. (2003). Venezuela: su enfermedad y su crisis actual. Cuestiones de América. Obtenido de http://www.cuestiones.ws/revista/n12/dic02-fuenmayor.htm 
García, J. (2016): Aproximación al estudio de la pobreza en México: Propuesta de política de estado contra la pobreza. Universidad Juárez Autónoma de Tabasco, Tabasco, México.

Hernández, R., Fernández, C., \& Baptista, P. (2014): Metodología de la investigación. MacGrawHill, México.

Herrera, F. (2013): Enfoques y políticas de desarrollo rural en México: Una revisión de su construcción institucional. Gestión y política pública. Disponible en http://www.scielo.org.mx/scielo.php?script=sci arttext\&pid $=\$ 1405-10792013000100004 \#$ nota

Huerta, H., y Chávez, M. (2003). Tres modelos de política económica en México durante los últimos sesenta años. Análisis Económico, 55-80.

Krugman, P., \& Wells, R. (2014): Macroeconomía. Reverté, México.

Miguel, A. (2004): Ciencia regional: Principios de economía y desarrollo. EUMED, España.

ONU. (2012). Desarrollo. Disponible en: https://www.un.org/es/sections/what-we-do/promote-sustainabledevelopment/

ONU. (25 de Septiembre de 2015): La Asamblea General adopta la Agenda 2030. Objetivos de Desarrollo Sostenible. Disponible en https://www.un.org/sustainabledevelopment/es/2015/09/la-asamblea-generaladopta-la-agenda-2030-para-el-desarrollo-sostenible/

Piketty, T. (2014): El capital en el siglo XXI. Fondo de Cultura Económica, México.

Pilonieta, C., y OCHOA, A. (2006). El desarrollo endógeno sustentable, una aproximación conceptual . Aprendiendo entorno al desarrollo endógeno, 21-34.

Portales, L. (2014): Los pobres como agentes de su desarrollo, la lucha contra la pobreza y la exclusión desde lo local. Revue Interventions Économiques, 51, 1-18. Disponible en https://journals.openedition.org/ interventionseconomiques/2172

Ramírez, A., Sánchez, J., \& García, A. (2004): El desarrollo sustentable: interpretación y análisis. Revista del Centro de Investigación. Universidad La Salle, vol. 6, núm. 21, julio-diciembre, 2004, 55-59. Disponible en https://www.redalyc.org/pdf/342/34202107.pdf

Roldán, N. (2017): Operan más de 5 mil programas sociales con gasto millonario, pero no logran disminuir la pobreza. Animal Político. Disponible en https://www.animalpolitico.com/2017/04/programas-socialespobreza

Samuelson, P., \& Nordhaus, W. (2010): Economía con aplicaciones para Latinoamérica. McGraw-Hill, México. Silva, I., \& Sandoval, C. (2012): Metodología para la elaboración de estrategias de desarrollo local. CEPAL, Chile. Zarta, P. (2018): La sustentabilidad o sostenibilidad: un concepto poderoso para la humanidad. Tábula Rasa, 28, 409-423. Disponible en http://www.scielo.org.co/pdf/tara/n28/1794-2489-tara-28-00409.pdf 